\title{
Dashboard Mechanisms for Online Marketplaces ${ }^{*}$
}

\author{
JASON D. HARTLINE, Northwestern University \\ ALECK JOHNSEN, Northwestern University \\ DENIS NEKIPELOV, University of Virginia \\ ONNO ZOETER, Booking.com
}

CCS Concepts: • Theory of computation $\rightarrow$ Algorithmic mechanism design.

\begin{abstract}
ACM Reference Format:
Jason D. Hartline, Aleck Johnsen, Denis Nekipelov, and Onno Zoeter. 2019. Dashboard Mechanisms for Online Marketplaces. In ACM EC '19: ACM Conference on Economics and Computation (EC '19), fune 24-28, 2019, Phoenix, AZ, USA. ACM, New York, NY, USA, 2 pages. https://doi.org/10.1145/3328526.3329653
\end{abstract}

This paper formalizes a theoretical study of bidding dashboards for the design of online markets. In these markets short-lived users arrive frequently and are matched with long-lived agents. Rarely in practice do market mechanisms, which allow the agents to bid to optimize their matching with the users, have truthtelling as an equilibrium strategy. ${ }^{1}$ The non-truthfulness of mechanisms for these markets is frequently the result of practical constraints; this paper is part of a small but growing literature on the design of these non-truthful mechanisms.

Two key challenges for the design of non-truthful mechanisms are (i) assisting the agents to find good strategies in these mechanisms and (ii) identifying mechanisms that have good outcomes when agents find good strategies. Bidding dashboards, which provide agents with market information relevant for bid optimization and are common in online marketplaces,${ }^{2}$ can provide a solution to both issues. This paper gives a formal study of dashboard mechanisms and identifies dashboards and accompanying mechanisms that lead agents to find good strategies and in which good strategies result in good outcomes.

The paper considers general environments for single-dimensional agents, i.e., with preferences given by a value for service and utility given by value times service probability minus payment. In winner-pays-bid mechanisms the agents report bids, the mechanism selects a set of agents to win, and all winners pay their bids. In all-pay mechanisms the agents report bids, the mechanism

\footnotetext{
*This work was done in part while the authors were at Booking.com. The first and second authors were supported in part by NSF CCF-1618502 and the third author by NSF CCF-1563708. The full paper can be found at: https://arxiv.org/abs/1905.05750 ${ }^{1}$ Such a matching is often the combination of a ranking of the agents by the market mechanism and the users' choice behavior. Examples of the agents in such mechanisms include, sellers posting a buy-it-now price on eBay, hoteliers choosing a commission percentage on Booking.com, or advertisers bidding in Google's ad auction. The first-two-examples are "winner pays bid" for the agents; none of these examples have truthtelling as an equilibrium.

${ }^{2}$ For example, Google provides a dashboard that forecasts click volume and cost per click as a function of advertisers' bids and Booking.com provides a visibility booster that forecasts percentage of increased clicks relative to base commission as a function of the commission percentage offered by the hotel for bookings.
}

Permission to make digital or hard copies of part or all of this work for personal or classroom use is granted without fee provided that copies are not made or distributed for profit or commercial advantage and that copies bear this notice and the full citation on the first page. Copyrights for third-party components of this work must be honored. For all other uses, contact the owner/author(s).

EC '19, fune 24-28, 2019, Phoenix, AZ, USA

(C) 2019 Copyright held by the owner/author(s).

ACM ISBN 978-1-4503-6792-9/19/06.

https://doi.org/10.1145/3328526.3329653 
selects a set of agents to win, and all agents pay their bids. For practical reasons, many markets are winner-pays-bid or all-pay. ${ }^{3}$

The motivating marketplaces for this work are ones where short-lived users perform a search or collection of searches in order to identify one or more long-lived agents with whom to transact. The market mechanism facilitates the user search by, for example, ranking the agents by a combination of perceived match quality for the user and the agents' willingness to pay for the match, henceforth, the agents' valuations. Strategically, the agents are in competition with each other for matching with the user. Viewing the user as a random draw from a population of users with varying tastes results in a stochastic allocation algorithm which maps the valuation profile of agents to the probabilities that a user selects each agent. This stochastic allocation algorithm is typically monotone in each agent's value and continuous [e.g., Athey and Nekipelov 2010].

A dashboard defines a bid allocation rule for each agent. Our dashboard mechanism assumes that the bids are best response to the published dashboard, i.e., that agents "follow the dashboard", and uses econometric inference to invert the profile of bids to determine the profile of values. The dashboard mechanism for a given dashboard and allocation algorithm is as follows:

(0) Publish a dashboard to each agent and solicit each agent's bid.

(1) Invert the bid of each agent, assuming it is in best response to the published dashboard, to obtain an inferred value for each agent.

(2) Execute the allocation algorithm on the inferred values to determine the outcome; charge each winner her bid (winner-pays-bid) or all agents their bids (all-pay).

Our main result is to identify dashboards where following the dashboard is not an assumption but near optimal strategy for agents. For online marketplaces, we define winner-pays-bid and all-pay dashboards for any sequence of allocation algorithms where following the dashboard for a sequence of values is approximately strategically equivalent to bidding those values in the sequential truthful mechanism corresponding to the sequence of allocation algorithms. Thus, the dashboard mechanism and the truthful mechanism are approximately strategically equivalent, i.e., their allocations are identical and their per-stage average differences in payments vanish with the number of stages.

Our dashboards can be implemented in the (blackbox) single-call model of mechanism design. In this model, an algorithm has been developed that is monotonic in each agent's value and obtains a good outcome. The mechanism's only access to the allocation algorithm is by live execution, i.e., where the outcome of the algorithm is implemented. Babaioff et al. [2010] introduced this model and showed that truthful mechanisms can be single-call implemented. We show that winner-pays-bid and all-pay dashboard mechanisms can be single-call implemented in online marketplaces.

The above approach to the design of online markets is simple and practical. Though we have specified the mechanism and framework for winner-pays-bid and all-pay mechanisms and singledimensional agents, the approach naturally generalizes to multi-dimensional environments and other kinds of mechanisms. It requires only that the preferences of the agents can be inferred from bids that are optimized for the dashboard. Winner-pays-bid, all-pay, and truthful formats can be mixed-and-matched, e.g., per the preferences of agents, in the same mechanism.

\section{REFERENCES}

Susan Athey and Denis Nekipelov. 2010. A structural model of sponsored search advertising auctions. In Sixth ad auctions workshop.

Moshe Babaioff, Robert D Kleinberg, and Aleksandrs Slivkins. 2010. Truthful mechanisms with implicit payment computation. In Proceedings of the 11th ACM conference on Electronic commerce. ACM, 43-52.

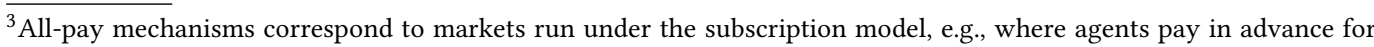
monthly for service but the quality of service depends on both the payment and the realized market conditions.
} 